\title{
Bipartite graphs whose squares are not chromatic-choosable
}

\author{
Seog-Jin Kim \\ Department of Mathematics Education \\ Konkuk University \\ Seoul, Korea \\ skim12@konkuk.ac.kr
}

\author{
Boram Park* \\ Department of Mathematics \\ Ajou University \\ Suwon, Korea \\ borampark@ajou.ac.kr
}

Submitted: May 2, 2014; Accepted: Feb 3, 2015; Published: Feb 25, 2015

Mathematics Subject Classifications: 05C15

\begin{abstract}
The square $G^{2}$ of a graph $G$ is the graph defined on $V(G)$ such that two vertices $u$ and $v$ are adjacent in $G^{2}$ if the distance between $u$ and $v$ in $G$ is at most 2. Let $\chi(H)$ and $\chi_{\ell}(H)$ be the chromatic number and the list chromatic number of $H$, respectively. A graph $H$ is called chromatic-choosable if $\chi_{\ell}(H)=\chi(H)$. It is an interesting problem to find graphs that are chromatic-choosable.

Motivated by the List Total Coloring Conjecture, Kostochka and Woodall (2001) proposed the List Square Coloring Conjecture which states that $G^{2}$ is chromaticchoosable for every graph $G$. Recently, Kim and Park showed that the List Square Coloring Conjecture does not hold in general by finding a family of graphs whose squares are complete multipartite graphs and are not chromatic choosable. It is a well-known fact that the List Total Coloring Conjecture is true if the List Square Coloring Conjecture holds for special class of bipartite graphs. Hence a natural question is whether $G^{2}$ is chromatic-choosable or not for every bipartite graph $G$.

In this paper, we give a bipartite graph $G$ such that $\chi_{\ell}\left(G^{2}\right) \neq \chi\left(G^{2}\right)$. Moreover, we show that the value $\chi_{\ell}\left(G^{2}\right)-\chi\left(G^{2}\right)$ can be arbitrarily large.
\end{abstract}

Keywords: Square of graph, chromatic-choosable, list chromatic number

\section{Introduction}

A proper $k$-coloring $\phi: V(G) \rightarrow\{1,2, \ldots, k\}$ of a graph $G$ is an assignment of colors to the vertices of $G$ so that any two adjacent vertices receive distinct colors. The chromatic number $\chi(G)$ of $G$ is the least $k$ such that there exists a proper $k$-coloring of $G$.

\footnotetext{
${ }^{*}$ Corresponding author: borampark@ajou.ac.kr
} 
A list assignment on $G$ is a function $L$ that assigns each vertex $v$ a set $L(v)$ which is a list of available colors at $v$. A graph $G$ is said to be $k$-choosable if for any list assignment $L$ such that $|L(v)| \geqslant k$ for every vertex $v$, there exists a proper coloring $\phi$ such that $\phi(v) \in L(v)$ for every $v \in V(G)$. The least $k$ such that $G$ is $k$-choosable is called the list chromatic number $\chi_{\ell}(G)$ of $G$. Clearly $\chi_{\ell}(G) \geqslant \chi(G)$ for every graph $G$.

A graph $G$ is called chromatic-choosable if $\chi_{\ell}(G)=\chi(G)$. It is an interesting problem to determine which graphs are chromatic-choosable. There are several famous conjectures that some classes of graphs are chromatic-choosable including the List Coloring Conjecture (see [5] for detail).

The line graph $L(G)$ of a graph $G$ is the graph such that $V(L(G))=E(G)$ and two vertices $x$ and $y$ are adjacent in $L(G)$ if and only if $x$ and $y$ are adjacent edges in $G$. The List Coloring Conjecture which asserts that $\chi_{\ell}(L(G))=\chi(L(G))$ for every graph $G$, where $L(G)$ is the line graph of $G$.

Borodin, Kostochka and Woodall [2] proposed the List Total Coloring Conjecture which asserts that $\chi_{\ell}(T(G))=\chi(T(G))$ for every graph $G$, where $T(G)$ is the total graph of $G$. Motivated by the List Total Coloring Conjecture, Kostochka and Woodall [8] proposed the List Square Coloring Conjecture which states that $G^{2}$ is chromatic-choosable for every graph $G$. It was noted in [8] that the List Total Coloring Conjecture is true if the List Square Coloring Conjecture is true. The List Square Coloring Conjecture has attracted a lot of attention and been cited in many papers related with coloring problems. Recently, Kim and Park [3] disproved the List Square Coloring Conjecture by finding a family of graphs whose squares are complete multipartite graphs with partite sets of unbounded size. Later, two different types of counterexamples to the List Square Coloring Conjecture have been known in $[4,7]$.

If $H$ is the graph obtained by placing a vertex in the middle of every edge of a graph $G$, then $H^{2}=T(G)$, where $T(G)$ is the total graph of $G$. Hence if the List Square Coloring Conjecture is true for a special class of bipartite graphs, then the List Total Coloring Conjecture is true. (see [8] for detail.)

On the other hand, all of the counterexamples in $[3,4,7]$ to the List Square Coloring Conjecture are not bipartite graphs. Hence a natural interesting question is whether $G^{2}$ is chromatic-choosable when $G$ is a bipartite graph. This question was raised by an anonymous referee and appeared in [3]. In this paper, we will give a bipartite graph $G$ such that $\chi_{\ell}\left(G^{2}\right) \neq \chi\left(G^{2}\right)$. Moreover, we show that the gap between $\chi_{\ell}\left(G^{2}\right)$ and $\chi\left(G^{2}\right)$ can be arbitrarily large for bipartite graphs $G$.

\section{Construction}

Let $[n]$ denote $\{1,2, \ldots, n\}$. A Latin square of order $n$ is an $n \times n$ array such that every cell contains an element of $[n]$ and every element of $[n]$ occurs exactly once in each row and each column. For a Latin square $L$ of order $n$, the element on the $i$ th row and the $j$ th column is denoted by $L(i, j)$. Two Latin squares $L_{1}$ and $L_{2}$ are orthogonal if for any $(i, j) \in[n] \times[n]$, there exists unique $(k, \ell) \in[n] \times[n]$ such that $L_{1}(k, \ell)=i$ and $L_{2}(k, \ell)=j$. 
From now on, we fix a prime number $n$ with $n \geqslant 3$. For $i \in[n-1]$, we define a Latin square $L_{i}$ of order $n$ by

$$
L_{i}(j, k)=j+i(k-1) \quad(\bmod n), \quad \text { for }(j, k) \in[n] \times[n] .
$$

Then it is easily checked (and well-known) that $L_{i}$ is a Latin square of order $n$ and $\left\{L_{1}, L_{2}, \ldots, L_{n-1}\right\}$ is a family of mutually orthogonal Latin squares of order $n$ (see page 252 in [9]). In Figure 1, $L_{1}$ and $L_{2}$ are orthogonal Latin squares defined in (1) for $n=3$.

$$
L_{1}=\begin{array}{|l|l|l|}
\hline 1 & 2 & 3 \\
\hline 2 & 3 & 1 \\
\hline 3 & 1 & 2 \\
\hline
\end{array} \quad L_{2}=\begin{array}{|l|l|l|}
\hline 1 & 3 & 2 \\
\hline 2 & 1 & 3 \\
\hline 3 & 2 & 1 \\
\hline
\end{array}
$$

Figure 1: Latin squares $L_{1}$ and $L_{2}$ of order 3 defined in (1).

Now we will construct a bipartite graph $G$ such that $G^{2}$ is not chromatic-choosable. First, we will describe briefly how to construct such bipartite graph $G$, and then will give a formal description in Construction 2.1.

\section{The procedure of the construction of $G$}

Step 1: For each prime number $n \geqslant 3$, we construct a graph $H_{n}$ with $2 n^{2}$ vertices as follows. For $k \in[n]$, let $P_{k}$ be the set of $n$ elements such that

$$
P_{k}=\left\{v_{k, 1}, v_{k, 2}, \ldots, v_{k, n}\right\},
$$

and for $i \in[n-1]$, let $Q_{i}$ be the set of $n$ elements such that

$$
Q_{i}=\left\{w_{i, 1}, w_{i, 2}, \ldots, w_{i, n}\right\},
$$

and let $S$ be the set of $n$ elements such that

$$
S=\left\{s_{1}, s_{2}, \ldots, s_{n}\right\} .
$$

Let $\left\{L_{1}, L_{2}, \ldots, L_{n-1}\right\}$ be the family of mutually orthogonal Latin squares of order $n$ obtained by (1). Graph $H_{n}$ is defined as follows:

$$
\begin{aligned}
& V\left(H_{n}\right)=\left(\cup_{k=1}^{n} P_{k}\right) \bigcup\left(\cup_{i=1}^{n-1} Q_{i}\right) \bigcup S, \\
& E\left(H_{n}\right)=\left(\bigcup_{i \in[n-1]} \bigcup_{j \in[n]}\left\{w_{i, j} v_{k, L_{i}(j, k)}: k \in[n]\right\}\right) \bigcup\left(\bigcup_{j \in[n]}\left\{s_{j} v_{k, j}: k \in[n]\right\}\right) .
\end{aligned}
$$

Let $T_{j}=\left\{v_{1, j}, v_{2, j}, \ldots, v_{n, j}\right\}$ for each $j \in[n]$. Note that for each vertex $w_{i, j}, N_{H_{n}}\left(w_{i, j}\right)=$ $\left\{v_{k, L_{i}(j, k)}: k \in[n]\right\}$, and $H_{n}$ is the graph obtained by removing the edges in

$$
\bigcup_{j \in[n]}\left\{x y: x, y \in T_{j}\right\}
$$


from the graph $G_{n}$ in [3] and adding vertices of $S$ and the edges of

$$
\bigcup_{j \in[n]}\left\{x s_{j}: x \in T_{j}\right\} \text {. }
$$

(Figure 2 is the case when $n=3$.)

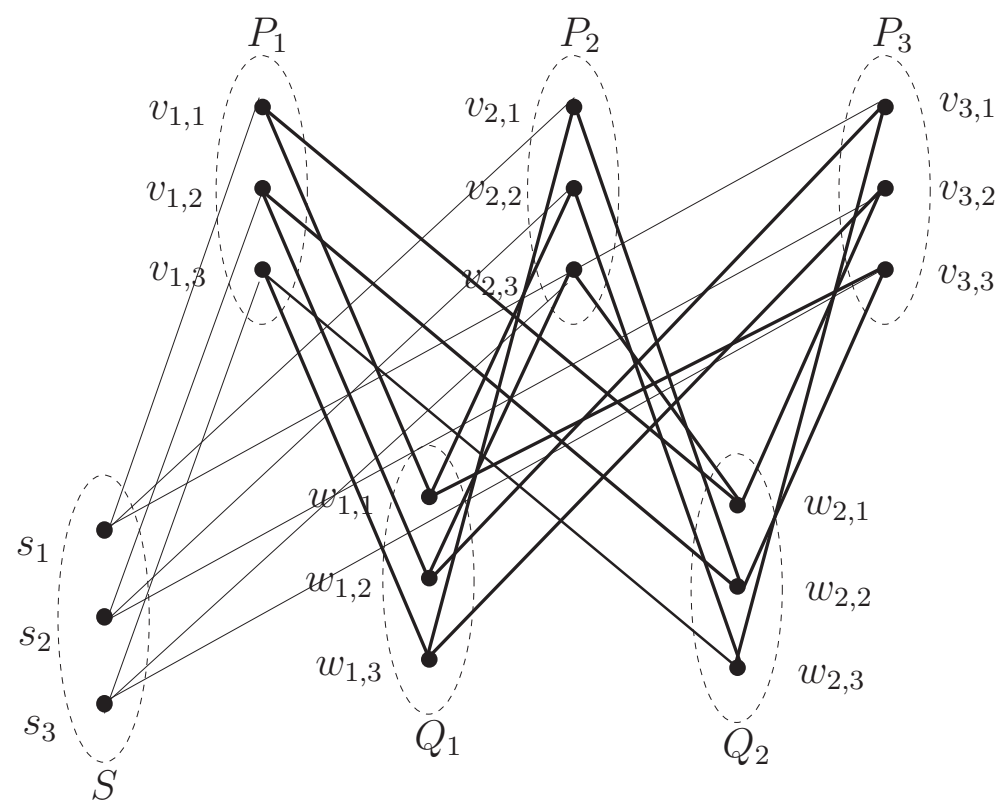

Figure 2: Graph $H_{n}$ when $n=3$ in Step 1. The bold edges induce the graph obtained by removing the edges in $\bigcup_{j \in[3]}\left\{x y: x, y \in T_{j}\right\}$ from graph $G_{3}$ in [3].

Given a graph $H$ and a vertex $v$ in $H$, duplicating $v$ means adding a new vertex $v_{0}$ and making it adjacent to all the neighbors of $v$ in $H$, but $v$ and $v_{0}$ are not adjacent. (See Figure 3 for an illustration.)
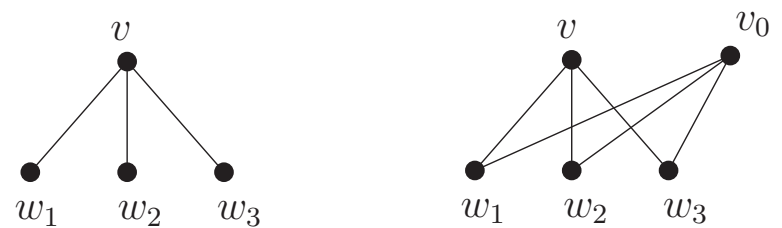

Figure 3: The graph on the right is obtained by duplicating the vertex $v$ of the graph on the left.

Step 2: Duplicate each vertex of $\cup_{k=1}^{n} P_{k}$ exactly $(n-1)$ times. For each vertex $v_{k, j}$, denote the $(n-1)$ copies of $v_{k, j}$ by $v_{k, j}^{2}, v_{k, j}^{3}, \ldots, v_{k, j}^{n}$, and denote the original vertex $v_{k, j}$ by $v_{k, j}^{1}$. 
Let $T_{l, m}=\left\{v_{1, m}^{l}, v_{2, m}^{l}, \ldots, v_{n, m}^{l}\right\}$ for each $m \in[n]$.

Step 3: For $i \in[n-1]$, let $R_{i}=\left\{u_{i, 1}, u_{i, 2}, \ldots, u_{i, n}\right\}$ be a set of new vertices. For each vertex $u_{i, j}$, make $u_{i, j}$ adjacent to all vertices in $\cup_{l=1}^{n} T_{l, L_{i}(j, l)}$. Note that the neighborhood of $u_{i, j}$ follows the same pattern of the neighborhood of $w_{i, j}$. For example, if $N_{H_{n}}\left(w_{i, j}\right)=$ $\left\{v_{k, L_{i}(j, k)}: k \in[n]\right\}$, then $N_{G}\left(u_{i, j}\right)=\cup_{k=1}^{n} T_{k, L_{i}(j, k)}$. Now, call the resulting graph $G$.

Figure 4 is an illustration of $G$ when $n=3$.

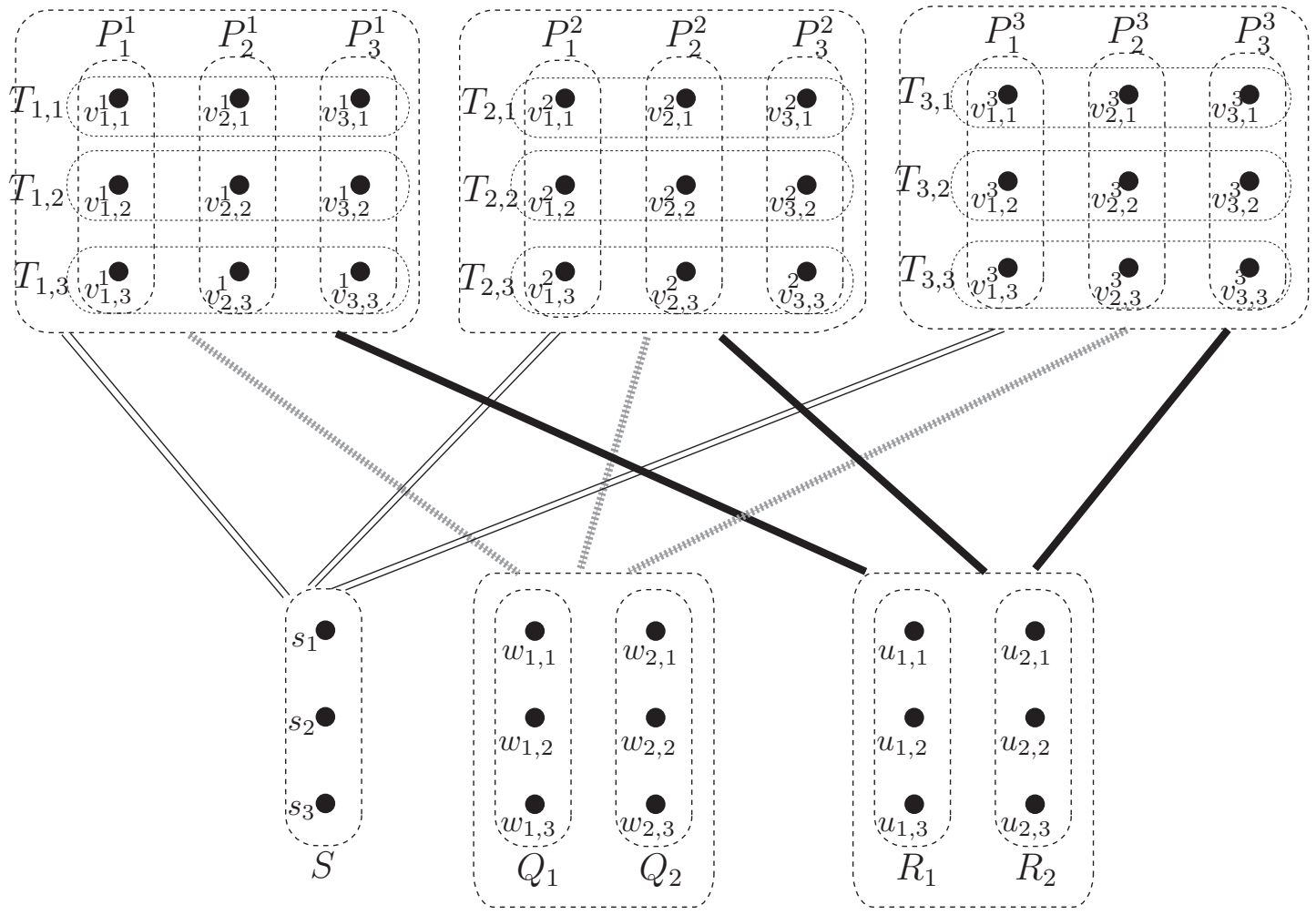

$\begin{cases}N_{G}\left(w_{1,1}\right)=\cup_{l=1}^{3}\left\{v_{1, \mathbf{1}}^{l}, v_{2, \mathbf{2}}^{l}, v_{3, \mathbf{3}}^{l}\right\}, & N_{G}\left(w_{2,1}\right)=\cup_{l=1}^{3}\left\{v_{1, \mathbf{1}}^{l}, v_{2, \mathbf{3}}^{l}, v_{3, \mathbf{2}}^{l}\right\}, \\ N_{G}\left(w_{1,2}\right)=\cup_{l=1}^{3}\left\{v_{1, \mathbf{2}}^{l}, v_{2, \mathbf{3}}^{l}, v_{3, \mathbf{1}}^{l}\right\}, & N_{G}\left(w_{2,2}\right)=\cup_{l=1}^{3}\left\{v_{1, \mathbf{2}}^{l}, v_{2, \mathbf{1}}^{l}, v_{3, \mathbf{3}}^{l}\right\}, \\ N_{G}\left(w_{1,3}\right)=\cup_{l=1}^{3}\left\{v_{1, \mathbf{3}}^{l}, v_{2, \mathbf{1}}^{l}, v_{3, \mathbf{2}}^{l}\right\}, & N_{G}\left(w_{2,3}\right)=\cup_{l=1}^{3}\left\{v_{1, \mathbf{3}}^{l}, v_{2, \mathbf{2}}^{l}, v_{3, \mathbf{1}}^{l}\right\},\end{cases}$

$\overline{-} \begin{cases}N_{G}\left(u_{1,1}\right)=T_{1, \mathbf{1}} \cup T_{2, \mathbf{2}} \cup T_{3, \mathbf{3}}, & N_{G}\left(u_{2,1}\right)=T_{1, \mathbf{1}} \cup T_{2, \mathbf{3}} \cup T_{3, \mathbf{2}}, \\ N_{G}\left(u_{1,2}\right)=T_{1, \mathbf{2}} \cup T_{2, \mathbf{3}} \cup T_{3, \mathbf{1}}, & N_{G}\left(u_{2,2}\right)=T_{1, \mathbf{2}} \cup T_{2, \mathbf{1}} \cup T_{3, \mathbf{3}}, \\ N_{G}\left(u_{1,3}\right)=T_{1, \mathbf{3}} \cup T_{2, \mathbf{1}} \cup T_{3, \mathbf{2}}, & N_{G}\left(u_{2,3}\right)=T_{1, \mathbf{3}} \cup T_{2, \mathbf{2}} \cup T_{2, \mathbf{1}},\end{cases}$

$=N_{G}\left(s_{1}\right)=\cup_{l=1}^{3} T_{l, \mathbf{1}}, \quad N_{G}\left(s_{2}\right)=\cup_{l=1}^{3} T_{l, \mathbf{2}}, \quad N_{G}\left(s_{3}\right)=\cup_{l=1}^{3} T_{l, \mathbf{3}}$.

Figure 4: Graph $G$ when $n=3$. 
Description of Figure 4: For each $l \in$ [3], the dotted line abbreviates adjacency between $P_{1}^{l} \cup P_{2}^{l} \cup P_{3}^{l}$ and $Q_{1} \cup Q_{2}$. For each $l \in[3]$, the bold line abbreviates adjacency between $P_{1}^{l} \cup P_{2}^{l} \cup P_{3}^{l}$ and $R_{1} \cup R_{2}$, and the doubled thin line abbreviates adjacency between the union of all $P_{k}^{l}$ 's and $S$. In $N_{G}\left(w_{i, j}\right)$ and $N_{G}\left(u_{i, j}\right)$, the bold subscripts are the $j$ th row of the Latin square $L_{i}$ which was defined in Figure 1, respectively. Note that for each $l \in[3]$ the subgraph induced by $P_{1}^{l} \cup P_{2}^{l} \cup P_{3}^{l} \cup Q_{1} \cup Q_{2} \cup S$ is isomorphic to graph $H_{3}$ in Figure 2 . Moreover, note that for any $1 \leqslant j_{1}, j_{2}, j_{3} \leqslant 3, P_{j_{1}}^{1} \cup P_{j_{2}}^{2} \cup P_{j_{3}}^{3} \cup R_{1} \cup R_{2} \cup S$ also induces a copy of $H_{3}$. (The indices $j_{1}, j_{2}, j_{3}$ are not necessarily distinct.)

The following is a formal description of the construction of $G$.

Construction 2.1. We construct a graph $G$ with $n\left(n^{2}+2 n-1\right)$ vertices as follows. For each $k, l \in[n]$, let $P_{k}^{l}$ be the set of $n$ elements such that

$$
P_{k}^{l}=\left\{v_{k, 1}^{l}, v_{k, 2}^{l}, \ldots, v_{k, n}^{l}\right\}
$$

and for each $i \in[n-1]$, let $Q_{i}$ be the set of $n$ elements such that

$$
Q_{i}=\left\{w_{i, 1}, w_{i, 2}, \ldots, w_{i, n}\right\}
$$

and for $i \in[n-1]$, let $R_{i}$ be the set of $n$ elements such that

$$
R_{i}=\left\{u_{i, 1}, u_{i, 2}, \ldots, u_{i, n}\right\}
$$

and let

$$
S=\left\{s_{1}, s_{2}, \ldots, s_{n}\right\}
$$

Let $\left\{L_{1}, L_{2}, \ldots, L_{n-1}\right\}$ be the family of mutually orthogonal Latin squares of order $n$ obtained by (1). For each $l, m \in[n]$, let

$$
T_{l, m}=\left\{v_{1, m}^{l}, v_{2, m}^{l}, \ldots, v_{n, m}^{l}\right\} .
$$

Now we define a graph $G$ as follows:

$$
\begin{aligned}
& V(G)=\left(\cup_{l=1}^{n} \cup_{k=1}^{n} P_{k}^{l}\right) \bigcup\left(\cup_{i=1}^{n-1} Q_{i}\right) \bigcup\left(\cup_{i=1}^{n-1} R_{i}\right) \cup S, \\
& E(G)=E_{1} \cup E_{2} \cup \cdots \cup E_{n} \cup E_{n+1} \cup E_{n+2},
\end{aligned}
$$

where

$$
\begin{aligned}
E_{l} & =\bigcup_{i \in[n-1]} \bigcup_{j \in[n]}\left\{w_{i, j} v_{k, L_{i}(j, k)}^{l}: k \in[n]\right\}, \text { for each } l \in[n], \\
E_{n+1} & =\bigcup_{i \in[n-1]} \bigcup_{j \in[n]}\left\{u_{i, j} y: y \in T_{l, L_{i}(j, l)} \text { for some } l \in[n]\right\}, \\
E_{n+2} & =\bigcup_{m \in[n]}\left\{s_{m} y: y \in T_{l, m} \text { for some } l \in[n]\right\} .
\end{aligned}
$$


By the definition of the graph $G$, it follows that

$$
\begin{aligned}
N_{G}\left(w_{i, j}\right) & =\bigcup_{l \in[n]}\left\{v_{1, L_{i}(j, 1)}^{l}, v_{2, L_{i}(j, 2)}^{l}, \ldots, v_{n, L_{i}(j, n)}^{l}\right\}, \\
N_{G}\left(u_{i, j}\right) & =T_{1, L_{i}(j, 1)} \cup T_{2, L_{i}(j, 2)} \cup \cdots \cup T_{n, L_{i}(j, n)}, \\
N_{G}\left(s_{m}\right) & =T_{1, m} \cup T_{2, m} \cup \cdots \cup T_{n, m} .
\end{aligned}
$$

For simplicity, for each $l \in[n]$, let $P^{l}=P_{1}^{l} \cup \cdots \cup P_{n}^{l}$ and let

$$
\begin{aligned}
& P=P^{1} \cup \cdots \cup P^{n}, \\
& Q=Q_{1} \cup \cdots \cup Q_{n-1}, \\
& R=R_{1} \cup \cdots \cup R_{n-1} .
\end{aligned}
$$

Let $K_{n \star r}$ denote the complete multipartite graph with $r$ partite sets in which each partite set has $n$ vertices. We will show that the subgraph of $G^{2}$ induced by $P$ is the complete multipartite graph $K_{n \star n^{2}}$ whose partite sets are $\left\{P_{k}^{l}: k, l \in[n]\right\}$.

For each $l \in[n]$, let $G_{l}$ be the subgraph of $G$ induced by $P^{l} \cup Q$. The following properties were obtained in Lemma 2.2 and Lemma 2.4 in [3].

Lemma 2.2. ([3]) For each $l \in[n], G_{l}$ satisfies the following properties.

(1) For any vertex $w \in Q$,

$$
\left|N_{G_{l}}(w) \cap P_{k}^{l}\right|=1, \text { for each } k \in[n] .
$$

(2) For any distinct vertices $w$ and $w^{\prime}$ in $Q$,

$$
\left|N_{G_{l}}(w) \cap N_{G_{l}}\left(w^{\prime}\right)\right| \leqslant 1 .
$$

(3) For any vertex $w \in Q$,

$$
\left|N_{G_{l}}(w) \cap T_{l, m}\right|=1, \text { for each } m \in[n] .
$$

(4) For any vertex $v \in P^{l}$,

$$
\left|N_{G_{l}}(v) \cap Q_{i}\right|=1, \text { for each } i \in[n-1] .
$$

From Lemma 2.2, we show the following lemmas.

Lemma 2.3. (1) For each $k, l \in[n], P_{k}^{l}$ is an independent set of $G^{2}$.

(2) For each $i \in[n-1], Q_{i}$ and $R_{i}$ are independent sets of $G^{2}$.

(3) The set $S$ is an independent set of $G^{2}$. 
Proof. Consider $P_{k}^{l}$ for some $k, l \in[n]$. Let $v, v^{\prime}$ be distinct vertices in $P_{k}^{l}$. We will show that $v$ and $v^{\prime}$ do not have a common neighbor. First, the vertices $v$ and $v^{\prime}$ do not have a common neighbor in $Q$ by (1) of Lemma 2.2. Next, $\left|N_{G}(u) \cap P_{k}^{l}\right|=1$ for any $u \in R$ by (3), and $\left|N_{G}(s) \cap P_{k}^{l}\right|=1$ for any $s \in S$ by (4). Hence $v$ and $v^{\prime}$ do not have a common neighbor in $R \cup S$. Thus $v$ and $v^{\prime}$ do not have a common neighbor in $G$, and consequently, $v$ and $v^{\prime}$ are not adjacent in $G^{2}$. Therefore, $P_{k}^{l}$ is an independent set in $G^{2}$.

Let $w$ and $w^{\prime}$ be any distinct vertices in $Q_{i}$. Suppose that the vertices $w$ and $w^{\prime}$ are adjacent in $G^{2}$. Since $G$ is a bipartite graph, they have a common neighbor $v$ in $P$. Then $v \in P^{l}$ for some $l$. Thus $w, w^{\prime} \in N_{G_{l}}(v) \cap Q_{i}$. But, by (4) of Lemma 2.2, $\left|N_{G_{l}}(v) \cap Q_{i}\right|=1$. This is a contradiction for the assumption that $w$ and $w^{\prime}$ are distinct. Therefore for each $i \in[n-1], Q_{i}$ is an independent set in $G^{2}$.

Let $u=u_{i, j}$ and $u^{\prime}=u_{i, j^{\prime}}$ be any distinct vertices in $R_{i}$. Suppose that the vertices $u$ and $u^{\prime}$ are adjacent in $G^{2}$. Then they have a common neighbor $v$ in $P$. Then $v \in$ $N_{G}(u) \cap N_{G}\left(u^{\prime}\right)$, and so by (3), $v \in T_{a, L_{i}(j, a)} \cap T_{b, L_{i}\left(j^{\prime}, b\right)}$ for some $a$ and $b$. Therefore, $a=b$ and $L_{i}(j, a)=L_{i}\left(j^{\prime}, a\right)$, which implies $j=j^{\prime}$ since $L_{i}$ is a Latin square. This is a contradiction. Thus for each $i \in[n-1], R_{i}$ is an independent set in $G^{2}$.

Moreover, it is clear that by (4), any two vertices in $S$ do not have a common neighbor in $G$, and so $S$ is an independent set of $G^{2}$.

Let $G^{2}\left[P^{l}\right]$ denote the subgraph of $G^{2}$ induced by $P^{l}$.

Lemma 2.4. For each $l \in[n], G^{2}\left[P^{l}\right] \cong K_{n * n}$ whose partite sets are $P_{1}^{l}, P_{2}^{l}, \ldots, P_{n}^{l}$.

Proof. The proof of Lemma 2.4 is similar to the proof of Lemma 2.8 in [3]. We include here for the sake of completeness. Take an integer $l \in[n]$. Note that $N_{G_{l}}(w) \subset P^{l}$ for each $w \in Q$ by the definition of $G_{l}$. First, note that $G^{2}\left[P^{l}\right]$ is isomorphic to a subgraph of $K_{n \star n}$, since for each $k, l \in[n], P_{k}^{l}$ is an independent set of $G^{2}\left[P^{l}\right]$ by Lemma 2.3. Let

$$
\mathcal{F}_{l}=\left\{G^{2}\left[N_{G}(w) \cap P^{l}\right]: w \in Q\right\} \cup\left\{G^{2}\left[N_{G}(s) \cap P^{l}\right]: s \in S\right\} .
$$

Note that for each $w \in Q, N_{G}(w) \cap P^{l}$ induces a complete graph $K_{n}$ in $G^{2}$, and for each $s \in S, N_{G}(s) \cap P^{l}$ induces a complete graph $K_{n}$ in $G^{2}$. Therefore $\mathcal{F}_{l}$ is a family of copies of $K_{n}$.

For any two vertices $w, w^{\prime} \in Q$, we have $\left|N_{G_{l}}(w) \cap N_{G_{l}}\left(w^{\prime}\right)\right| \leqslant 1$ by (2) of Lemma 2.2 and so $\left|N_{G}(w) \cap N_{G}\left(w^{\prime}\right) \cap P^{l}\right| \leqslant 1$. This implies that $G^{2}\left[N_{G}(w) \cap P^{l}\right]$ and $G^{2}\left[N_{G}\left(w^{\prime}\right) \cap P^{l}\right]$ are edge-disjoint. Note that $N_{G}\left(s_{m}\right) \cap P^{l}=T_{l, m}$ for each $m \in[n]$. Thus $T_{l, m} \cap T_{l, m^{\prime}}=\emptyset$ if $m \neq m^{\prime}$. This implies that if $s \neq s^{\prime}$, then $G^{2}\left[N_{G}(s) \cap P^{l}\right]$ and $G^{2}\left[N_{G}\left(s^{\prime}\right) \cap P^{l}\right]$ are edge-disjoint. Next, by (3) of Lemma 2.2, for each $m \in[n],\left|N_{G_{l}}(w) \cap T_{l, m}\right|=1$. Thus $\left|N_{G}(w) \cap P^{l} \cap T_{l, m}\right|=1$. This implies that $G^{2}\left[N_{G}(w) \cap P^{l}\right]$ and $G^{2}\left[N_{G}(s) \cap P^{l}\right]$ are edge-disjoint. Therefore any two cliques in $\mathcal{F}_{l}$ are edge-disjoint.

In addition, $\left|\mathcal{F}_{l}\right|=|Q|+|S|=n(n-1)+n=n^{2}$. Thus $\mathcal{F}_{l}$ is a family of $n^{2}$ pairwise edge-disjoint cliques of size $n$ in $G^{2}\left[P^{l}\right]$. It follows that

$$
\left|E\left(K_{n * n}\right)\right| \geqslant\left|E\left(G^{2}\left[P^{l}\right]\right)\right| \geqslant n^{2} \times\left(\begin{array}{l}
n \\
2
\end{array}\right)=\left|E\left(K_{n * n}\right)\right| .
$$

Hence $G^{2}\left[P^{l}\right] \cong K_{n * n}$ for each $l \in[n]$, since $G^{2}\left[P^{l}\right]$ is isomorphic to a subgraph of $K_{n * n}$. 
To show that $G^{2}[P] \cong K_{n \star n^{2}}$, it remains to show the following lemma.

Lemma 2.5. For any distinct $s, t \in[n]$, for any $v \in P^{s}$ and $v^{\prime} \in P^{t}, v$ and $v^{\prime}$ are adjacent in $G^{2}$.

Proof. Let $v \in P^{s}$ and $v^{\prime} \in P^{t}$ be any vertices with $s \neq t$. Then $v \in T_{s, a}$ and $v^{\prime} \in T_{t, b}$ for some $a, b \in[n]$. If $a=b$, then $T_{s, a} \cup T_{t, b} \subset N_{G}\left(s_{a}\right)$ and so $v$ and $v^{\prime}$ have a common neighbor $s_{a}$ in $G$. Hence $v v^{\prime} \in E\left(G^{2}\right)$.

We will show that if $a \neq b$, then there exist $i \in[n-1]$ and $j \in[n]$ such that $L_{i}(j, s)=a$ and $L_{i}(j, t)=b$ for fixed $s$ and $t$. Note that if $a \neq b$ and $s \neq t$, then there exist $i$ and $j$ satisfying the following equations.

$$
\begin{aligned}
j+i(s-1) & \equiv a & & (\bmod n) \\
j+i(t-1) & \equiv b & & (\bmod n) .
\end{aligned}
$$

Thus from (1), we know that there exist $i \in[n-1]$ with $L_{i}(j, s)=a$, and $j \in[n]$ with $L_{i}(j, t)=b$. Note that by (3), we have $T_{s, L_{i}(j, s)} \cup T_{t, L_{i}(j, t)} \subset N_{G}\left(u_{i, j}\right)$. Therefore $T_{s, a} \cup T_{t, b} \subseteq$ $N_{G}\left(u_{i, j}\right)$, and so $v$ and $v^{\prime}$ have a common neighbor $u_{i, j}$ in $G$. Hence $v v^{\prime} \in E\left(G^{2}\right)$.

By Lemmas 2.3, 2.4 and 2.5, the following theorem holds.

Theorem 2.6. If $G$ is the graph defined in Construction 2.1, then $G^{2}[P] \cong K_{n \star n^{2}}$ whose partite sets are the $P_{k}^{l}$ 's.

The following lower bound on the list chromatic number of a complete multipartite graph was obtained in [10].

Theorem 2.7. (Theorem 4, [10]) For a complete multipartite graph $K_{n \star r}$ with $n, r \geqslant 2$,

$$
\chi_{\ell}\left(K_{n \star r}\right)>(n-1)\left\lfloor\frac{2 r-1}{n}\right\rfloor .
$$

Consequently, we obtain that $\chi_{\ell}(G)>\chi(G)$ by the following theorem.

Theorem 2.8. For each prime $n \geqslant 3$, if $G$ is the graph defined in Construction 2.1, then

$$
\chi_{\ell}\left(G^{2}\right)-\chi\left(G^{2}\right)>n^{2}-6 n+3 .
$$

Proof. It is clear that $\chi\left(G^{2}\right) \leqslant n^{2}+2 n-1$ by Lemma 2.3. On the other hand, by Theorems 2.6 and 2.7,

$$
\chi_{\ell}\left(G^{2}\right) \geqslant \chi_{\ell}\left(K_{n \star n^{2}}\right)>(n-1)\left\lfloor\frac{2 n^{2}-1}{n}\right\rfloor \geqslant 2(n-1)^{2} .
$$

Thus

$$
\chi_{\ell}\left(G^{2}\right)-\chi\left(G^{2}\right)>2(n-1)^{2}-\left(n^{2}+2 n-1\right)=n^{2}-6 n+3 .
$$

Remark 2.9. Note that for any prime $n \geqslant 7$, we have $\chi_{\ell}\left(G^{2}\right)-\chi\left(G^{2}\right)>n^{2}-6 n+3>0$. Thus from Theorem 2.8, there exists a bipartite graph $G$ such that $G^{2}$ is not chromaticchoosable. Furthermore, since there are infinitely many primes, the gap $\chi_{\ell}\left(G^{2}\right)-\chi\left(G^{2}\right)$ can be arbitrarily large. Note that for large $n$ there exists a constant $c$ such that $\chi_{\ell}\left(G^{2}\right) \geqslant$ $c \chi\left(G^{2}\right) \log \left(\chi\left(G^{2}\right)\right)$ by applying the well-known result of Alon [1]. 


\section{Further Discussion}

Note that from (4) of Lemma 2.2, each vertex $v$ in $P$ has exactly one neighbor in each of $Q_{i}, R_{j}$, and $S$, respectively. Thus, if $G$ is the bipartite graph defined in Construction 2.1 for prime number $n$, then $d_{G}(x)=2 n-1$ for each $x \in P$ and $d_{G}(y)=n^{2}$ for each $y \in Q \cup R \cup S$. Hence from Theorem 2.8, if $G$ is the bipartite graph defined in Construction 2.1 for $n=7$, then $G^{2}$ is not chromatic-choosable and every vertex of one partite set of $G$ has degree 13. Note that the List Total Coloring Conjecture is true if the List Square Coloring Conjecture holds for bipartite graphs such that every vertex of one partite set has degree at most 2. Thus, it would be interesting to answer the following questions.

Question 3.1. If $G$ is a bipartite graph such that every vertex of one partite set has degree at most 2 , then is it true that $\chi_{\ell}\left(G^{2}\right)=\chi\left(G^{2}\right)$ ?

Question 3.2. If the answer to Question 3.1 is yes, then what is the largest $k$ such that $G^{2}$ is chromatic-choosable for every bipartite graph $G$ with a partite set in which each vertex has degree at most $k$ ?

We already mentioned that there is a bipartite graph $G$ such that every vertex of one partite set of $G$ has degree 13 and $G^{2}$ is not chromatic-choosable. Thus if the answer to Question 3.1 is yes (or the List Total Coloring Conjecture is true), then the $k$ in Question 3.2 must be less than 13 .

On the other hand, if we apply the 'duplication idea' in Step 2 in the procedure of the construction of $G$ repeatedly, then we can obtain a bipartite graph $\mathcal{G}$ such that every vertex of one partite set of $\mathcal{G}$ has degree 7 and $\mathcal{G}^{2}$ is not chromatic-choosable. This implies that the integer $k$ in Question 3.2 must be less than 7 .

We will describe briefly how to construct such bipartite graph $\mathcal{G}$. Let $G$ be the graph in Construction 2.1 when $n=3$. Now we duplicate each vertex of $P$ exactly 2 times. For each vertex $v_{k, j}^{l}$, we denote its copies by $v_{k, j}^{\prime l}$ and $v_{k, j}^{\prime \prime l}$. Let $P^{\prime}$ denote the set of the first copied vertices $v_{k, j}^{\prime l}$, and let $P^{\prime \prime}$ denote the set of the second copied vertices $v_{k, j}^{\prime \prime l}$. For each $h \in[3]$, let $\mathcal{T}_{1, h}=T_{1, h} \cup T_{2, h} \cup T_{3, h}$, that is,

$$
\mathcal{T}_{1, h}=\left\{v_{1, h}^{1}, v_{2, h}^{1}, v_{3, h}^{1}, v_{1, h}^{2}, v_{2, h}^{2}, v_{3, h}^{2}, v_{1, h}^{3}, v_{2, h}^{3}, v_{3, h}^{3}\right\} .
$$

In addition, let the two copies corresponding to $\mathcal{T}_{1, h}$ be denoted as follows:

$$
\begin{aligned}
& \mathcal{T}_{2, h}=\left\{v_{1, h}^{\prime 1}, v_{2, h}^{\prime 1}, v_{3, h}^{\prime 1}, v_{1, h}^{\prime 2}, v_{2, h}^{\prime 2}, v_{3, h}^{\prime 2}, v_{1, h}^{\prime 3}, v_{2, h}^{\prime 3}, v_{3, h}^{\prime 3}\right\}, \\
& \mathcal{T}_{3, h}=\left\{v^{\prime \prime 1}{ }_{1, h}^{1}, v^{\prime \prime}{ }_{2, h}^{1}, v^{\prime \prime 1}{ }_{3, h}, v^{\prime \prime 2}{ }_{1, h}^{2}, v^{\prime \prime 2}{ }_{2, h}, v^{\prime \prime 2}{ }_{3, h}^{2}, v^{\prime \prime 3}{ }_{1, h}, v^{\prime \prime}{ }_{2, h}^{3}, v^{\prime \prime 3}{ }_{3, h}^{3}\right\} .
\end{aligned}
$$

Next, we introduce 6 new vertices of $B_{1} \cup B_{2}$ where $B_{1}=\left\{b_{1,1}, b_{1,2}, b_{1,3}\right\}$ and $B_{2}=$ $\left\{b_{2,1}, b_{2,2}, b_{2,3}\right\}$, in which the neighborhood of each vertex $b_{i, j} \in B_{1} \cup B_{2}$ follows the same pattern of the neighborhood of $w_{i, j}$ (similar to Step 3 in the procedure of the construction of $G$ ). More precisely, $N_{\mathcal{G}}\left(b_{i, j}\right)=\cup_{k=1}^{3} \mathcal{T}_{k, L_{i}(j, k)}$ for each $b_{i, j}$, where $\mathcal{G}$ is the resulting graph. See Figure 5 for an illustration and its description is below. 




$$
\begin{array}{ll}
N_{\mathcal{G}}\left(b_{1,1}\right)=\mathcal{T}_{1, \mathbf{1}} \cup \mathcal{T}_{2, \mathbf{2}} \cup \mathcal{T}_{3, \mathbf{3}}, & N_{\mathcal{G}}\left(b_{2,1}\right)=\mathcal{T}_{1, \mathbf{1}} \cup \mathcal{T}_{2, \mathbf{3}} \cup \mathcal{T}_{3, \mathbf{2}} \\
N_{\mathcal{G}}\left(b_{1,2}\right)=\mathcal{T}_{1, \mathbf{2}} \cup \mathcal{T}_{2, \mathbf{3}} \cup \mathcal{T}_{3, \mathbf{1}}, & N_{\mathcal{G}}\left(b_{2,2}\right)=\mathcal{T}_{1, \mathbf{2}} \cup \mathcal{T}_{2, \mathbf{1}} \cup \mathcal{T}_{3, \mathbf{3}} \\
N_{\mathcal{G}}\left(b_{1,3}\right)=\mathcal{T}_{1,3} \cup \mathcal{T}_{2, \mathbf{1}} \cup \mathcal{T}_{3, \mathbf{2}}, & N_{\mathcal{G}}\left(b_{2,3}\right)=\mathcal{T}_{1, \mathbf{3}} \cup \mathcal{T}_{2, \mathbf{2}} \cup \mathcal{T}_{2, \mathbf{1}}
\end{array}
$$

Figure 5: Graph $\mathcal{G}$

Description of Figure 5: The sets $P^{\prime}$ and $P^{\prime \prime}$ are copies of $P$, and the bold line abbreviates adjacency between $P$ and $S \cup Q \cup R$. Each of three $P \cup S \cup Q \cup R, P^{\prime} \cup S \cup Q \cup R$, $P^{\prime \prime} \cup S \cup Q \cup R$ induces a graph isomorphic to graph $G$ in Figure 4. Like as $N_{G}\left(w_{i, j}\right)$ and $N_{G}\left(u_{i, j}\right)$, in $N_{\mathcal{G}}\left(b_{i, j}\right)$, the bold subscripts are the $j$ th row of the Latin square $L_{i}$ which was defined in Figure 1.

Then, the resulting graph $\mathcal{G}$ is a bipartite graph with partite sets $X=P \cup P^{\prime} \cup P^{\prime \prime}$ and $Y=S \cup Q \cup R \cup B_{1} \cup B_{2}$. Note that each vertex $x \in X$ has degree 7 and each vertex $y \in Y$ has degree 27. Then for each $k, l \in[3]$, we can see that $P_{k}^{l}=\left\{v_{k, 1}^{l}, v_{k, 2}^{l}, v_{k, 3}^{l},\right\}$ is an independent set in $\mathcal{G}^{2}$ and each of its corresponding copies $P_{k}^{\prime l}$ and $P_{k}^{\prime \prime l}$ is also an independent set in $\mathcal{G}^{2}$. In addition, each of $S, Q_{1}, Q_{2}, R_{1}, R_{2}, B_{1}, B_{2}$ is an independent set in $\mathcal{G}^{2}$. Thus we know that $\mathcal{G}^{2}$ is a multipartite graph with 34 partite sets. Therefore $\chi\left(\mathcal{G}^{2}\right) \leqslant 34$. Moreover, we can easily check that the subgraph of $\mathcal{G}^{2}$ induced by $X$ is the complete multipartite graph $K_{3 \star 27}$, and so $\chi_{\ell}\left(\mathcal{G}^{2}\right) \geqslant \chi_{\ell}\left(K_{3 \star 27}\right)=\left\lceil\frac{4 \times 27-1}{3}\right\rceil=36$ (see [6]). Thus $\mathcal{G}^{2}$ is not chromatic-choosable.

Remark 3.3. In general, for each prime number $n$, if we apply this duplication idea $d$ times to the graph $H_{n}$ in the Construction 2.1, then we have a bipartite graph whose square is a multiparite graph with $n^{d}+d(n-1)+1$ partite sets, containing a complete 
multiparitite graph $K_{n \star n^{d}}$. Through this way, we can also construct many bipartite graphs whose square are not chromatic-choosable.

\section{Acknowledgements}

The first author (S.-J. Kim) was supported by the National Research Foundation of Korea(NRF) grant funded by the Korea government (MEST)(No. 2011-0009729), and the second author's work was (partially) supported by the new faculty research fund of Ajou University (S-2014-G0001-00293).

\section{References}

[1] N. Alon, Choice numbers of graphs: a probabilistic approach., Combin. Probab. Comput., 1(2):107-114, 1992.

[2] O. V. Borodin, A. V. Kostochka and D. R. Woodall, List edge and list total colourings of multigraphs, J. Combin. Theory Ser. B, 71:184-204, 1997.

[3] S.-J. Kim and B. Park, Counterexamples to the List Square Coloring Conjecture, J. Graph Theory, in press.

[4] S.-J. Kim, Y. S. Kwon, and B. Park, Chromatic-choosability of the power of graphs, Discrete Appl. Math., 180:120-125, 2015.

[5] T. R. Jensen and B. Toft, Graph Coloring Problems, Wiley-Interscience, New York, 1995.

[6] H. A. Kierstead, On the choosability of complete multipartite graphs with part size three, Discrete Math., 211:255-259, 2000.

[7] N. Kosar, S. Petrickova, B. Reiniger, and E. Yeager, A note on list-coloring powers of graphs, Discrete Math., 332:10-14, 2014.

[8] A. V. Kostochka and D. R. Woodall, Choosability conjectures and multicircuits, Discrete Math., 240:123-143, 2001.

[9] J. H. van Lint and R. M. Wilson, A Course in Combinatorics, Cambridge University Press, 1992.

[10] T. Vetrík, List coloring of complete multipartite graphs, Discuss. Math. Graph Theory, 32:31-37, 2012. 